\title{
Two extensions of Thurston's spectral theorem for surface diffeomorphisms
}

\author{
Anders Karlsson*
}

August 3, 2018

\begin{abstract}
Thurston obtained a classification of individual surface homeomorphisms via the dynamics of the corresponding mapping class elements on Teichmüller space. In this paper we present certain extended versions of this, first, to random products of homeomorphisms and second, to holomorphic self-maps of Teichmüller spaces.
\end{abstract}

\section{Introduction}

Let $M$ be an oriented closed surface of genus $g \geq 2$. Let $\mathcal{S}$ denote the isotopy classes of simple closed curves on $M$ not isotopically trivial. For a Riemannian metric $\rho$ on $M$ and aclosed curve $\beta$, let $l_{\rho}(\beta)$ be the infimum of the length of curves isotopic to $\beta$.

In a seminal preprint from 1976 [T88, Thurston classified surface diffeomorphisms as being isotopic either to a periodic one, or else reducible or pseudo-Anosov. A version of this was obtained earlier in a series of papers by Nielsen $\mathrm{N}$, N44. Using the theory of foliations of surfaces, Thurston showed the following consequence; the proof is worked out in exposé 11 of [FLP79, Théorème Spectral]:

Theorem 1. ([T88, Theorem 5]) For any diffeomorphism $f$ of $M$, there is a finite set $1 \leq$ $\lambda_{1}<\lambda_{2}<\ldots<\lambda_{K}$ of algebraic integers such that for any $\alpha \in \mathcal{S}$ there is a $\lambda_{i}$ such that for any Riemannian metric $\rho$,

$$
\lim _{n \rightarrow \infty} l_{\rho}\left(f^{n} \alpha\right)^{1 / n}=\lambda_{i} .
$$

The map $f$ is isotopic to a pseudo-Anosov map iff $K=1$ and $\lambda_{1}>1$.

This statement is analogous to the dynamical behaviour of linear maps $A$ of finite dimensional vector spaces: the $\operatorname{limits}_{\lim _{n \rightarrow \infty}}\left\|A^{n} v\right\|^{1 / n}$ exist for every vector $v$, as is immediate from the Jordan normal form. In this note we will obtain a few extensions of Theorem 1 First:

Theorem 2. For any integrable ergodic cocycle $f_{n}=g_{n} g_{n-1} \ldots g_{1}$ of orientation-preserving homeomorphisms of $M$, there are almost surely a constant $\lambda \geq 1$ and a (random) measured foliation $\mu$ such that for any $\alpha \in \mathcal{S}$ with $i(\mu, \alpha)>0$ and Riemannian metric $\rho$,

$$
\lim _{n \rightarrow \infty} l_{\rho}\left(f_{n} \alpha\right)^{1 / n}=\lambda .
$$

* Supported in part by Institut Mittag-Leffler (Djursholm, Sweden) and the Swiss NSF grant 200021 $132528 / 1$. 
Let $\mathcal{T}$ be the Teichmüller space of $M$ and for $x \in \mathcal{T}$, the corresponding hyperbolic length of $\alpha \in \mathcal{S}$ is denoted by $l_{x}(\alpha)$. The previous theorem is a direct consequence of the more precise statement:

Theorem 3. In the setting of the previous theorem, we have almost surely that for any $x \in \mathcal{T}$, there is an explicit constant $C(\mu, x)>0$ such that for any $\epsilon>0$ there is a number $N$ for which

$$
C(\mu, x) i(\mu, \alpha)(\lambda-\epsilon)^{n} \leq l_{x}\left(f_{n} \alpha\right) \leq l_{x}(\alpha)(\lambda+\epsilon)^{n}
$$

holds for any $\alpha \in \mathcal{S}$ and any $n>N$.

Notice in particular the uniformity in $\alpha$ and the appearance of $i(\mu, \alpha)$.

The mapping class group $M C G(M)$ is the group of isotopy classes of orientation-preserving homeomorphisms (or diffeomorphisms) of $M$ :

$$
M C G(M)=\text { Homeo }^{+}(M) / \text { Homeo }_{0}(M),
$$

which acts by automorphisms of $\mathcal{T}(M)$. Thus every random product of homeomorphism gives rise to a random product of mapping classes and acts on $\mathcal{T}$. Kaimanovich-Masur [KM96] studied random walks on $M C G$. They proved that if the support of the random walk measure generates a non-elementary subgroup, then a.e. trajectory converges to points in the set of uniquely ergodic foliations of $\mathcal{P} \mathcal{M F}$. Taking this into account, we can deduce:

Corollary 4. Let $f_{n}=g_{n} g_{n-1} \ldots g_{1}$ be a product of random homeomorphisms where $g_{i}$ are chosen independently and distributed with a probability measure of finite first moment and that generates a subgroup containing two independent pseudo-Anosov maps. Then there is a number $\lambda>1$ such that a.s. for any $\alpha \in \mathcal{S}$ and metric $\rho$

$$
\lim _{n \rightarrow \infty} l_{\rho}\left(f_{n} \alpha\right)^{1 / n}=\lambda .
$$

This can be viewed as analogous to a well-known theorem of Furstenberg and to Oseledets' multiplicative ergodic theorem for random products of matrices. Information about these results and other references to the vast literature on random dynamical systems can be found in A98].

Given a complex structure $x$ on $M$, the extremal length of an isotopy class of curve $\alpha$ is

$$
\operatorname{Ext}_{x}(\alpha)=\sup _{\rho \in[x]} \frac{l_{\rho}(\alpha)^{2}}{\operatorname{Area}(\rho)}
$$

where the supremum is taken over all metrics in the conformal class of $x$. Miyachi [Mi08] noted that a normalized version of the extremal length function, denoted $E_{P}$, extends continuously to the whole Gardiner-Masur compactification, see section 3 below for more details. A boundary point $P$ is called uniquely ergodic if $E_{P}(\beta)>0$ for all $\beta \in \mathcal{S}$.

By a theorem of Royden from 1971, later extended by Earle-Kra to surfaces $M$ with punctures, the mapping class group, with some lower genus exceptions, is isomorphic to the complex automorphism group of $\mathcal{T}(\mathrm{M})$. Here is a statement about more general holomorphic selfmaps, thus in a sense providing a certain extension of Theorem 1 .

Theorem 5. Let $f: \mathcal{T} \rightarrow \mathcal{T}$ be a holomorphic map and $x \in \mathcal{T}$. Then there is a number $\lambda \geq 1$ and a point $P$ in the Gardiner-Masur compactification such that for all $n \geq 1$ and any curve $\beta \in \mathcal{S}$,

$$
\operatorname{Ext}_{f^{n} x}(\beta) \geq\left(\inf _{\alpha} \frac{\operatorname{Ext}_{x}^{1 / 2}(\alpha)}{E_{P}(\alpha)}\right)^{2} E_{P}(\beta)^{2} \lambda^{n}
$$


and, provided that $E_{P}(\beta)>0$,

$$
\operatorname{Ext}_{f^{n} x}(\beta)^{1 / n} \rightarrow \lambda
$$

The following can be seen as a weak generalization of the Nielsen-Thurston classification of mapping classes to general holomorphic self-maps of Teichmüller spaces:

Theorem 6. Let $f: \mathcal{T} \rightarrow \mathcal{T}$ be a holomorphic map. Then either every orbit in $\mathcal{T}$ is bounded, or every orbit leaves every compact set and there are associated points $P$ in the GardinerMasur boundary. If $P$ is uniquely ergodic, then it is unique and every orbit converges to this point in either compactification and for some $\lambda \geq 1$, any $\alpha \in \mathcal{S}$ and any $x \in \mathcal{T}$

$$
\operatorname{Ext}_{f^{n} x}(\alpha)^{1 / n} \rightarrow \lambda \text { and } \inf _{\alpha} \frac{\operatorname{Ext}_{f(x)}^{1 / 2}(\alpha)}{E_{P}(\alpha)} \geq \lambda \inf _{\alpha} \frac{\operatorname{Ext}_{x}^{1 / 2}(\alpha)}{E_{P}(\alpha)} .
$$

This classifies $f$ as either having bounded orbits or else having certain associated boundary points $P$ ("reducible" vs pseudo-Anosov depending on the nature of $P$ ). This is reminiscent of the Wolff-Denjoy theorem in complex dynamics that, together with a theorem of Fatou, classifies holomorphic self-maps of the unit disk. The $P$ s can informally be regarded as "virtual fixed points at infinity". Examples of important holomorphic self-maps beyond the automorphisms are the Thurston skinning map in three-dimensional topology and the Thurston pull-back maps in complex dynamics, see [Mc90, Pi01, S11] for more details.

\section{Method of proof:}

For the ergodic statements, the techniques of Ledrappier and myself [KaL11 are being employed, although extended to asymmetric metrics, such as Thurston's Lipschitz metric. Together with a comparison with Teichmüller's metric, and, crucially, the new insights about horofunctions by Cormac Walsh W11, this leads to the main result Theorem 3. The proof of the corollary relies in addition on KM96.

For Theorems 5 and 6 , the starting points is the well-known fact that the Techmüller metric coincides with the Kobayashi metric, which implies that holomorphic maps are 1-Lipschitz in this metric (this is the only way in which the holomorphy is being used), after that the proof uses recent results on horofunctions [Mi08, LS10, Mi11] and Ka01.

\section{Further comments:}

- While Nielsen studied lifts of the diffeomorphisms to the hyperbolic disk and boundary circle, Thurston instead compactified $\mathcal{T}$ in a natural way, by adding $\mathcal{P} \mathcal{M F}$, and applied Brouwer's fixed point theorem. Bers gave an alternative approach using more classical theory, in particular the Teichmüller metric [B78]. For the spectral theorem (Theorem 11) Thurston used foliation theory, see [FLP79] and also [FMa12, §14.5]. Our approach here instead uses two metrics on $\mathcal{T}$ : Thurston's and Teichmüller's. In this context it might be useful to point out that the recent paper [LPST11] contians a study parallel to Bers' paper but for the Thurston metric.

- Following the work of Kaimanovich-Masur [M95, KM96] it is natural to wonder about socalled ray approximation, see [K00] where this problem is mentioned. The first result in this direction is a paper of Duchin [D05] which established ray approximation in the Teichmüller metric for times when the ray visits the thick part. Recently there is an elegant argument by Tiozzo [Ti12] removing the thick part requirement, this was first obtained for finitely supported measures and then later (after the present paper was completed) under finite first moment. This provides another approach to Corollary 4, Ray approximation for Weil-Petersson geodesics in the more general ergodic setting follows from the arguments in a 
paper by Margulis and myself, see [KaL06]. A recent analysis of the harmonic measure can be found in Ga09.

- During the last few years there has been great progress in proving that almost every element in $M C G$ is pseudo-Anosov thanks to the works of Maher, Rivin, Kowalski, and LubotzkyMeiri, see e.g. Ma11. It is perhaps interesting to compare this with Corollary 4 above which states that random walk trajectories eventually look pseudo-Anosov from the perspective of Theorem 1.

- Looking at Theorem 1 or Oseledets' theorem in the linear case, one might hope for a more precise statement than Theorem [2. There are however some obvious obstructions to keep in mind. The exponent $\lambda$ can in general take any real value $\geq 1$ since one can take an appropriately asymmetric measure on a single pseudo-Anosov and its inverse. Moreover, for predicting which $\alpha$ grows exponentially in length, one should bear in mind the case of a coboundary $g(x)=f(\omega) f(T \omega)^{-1}$ and that the cocycle might consist of reducible maps with respect to one and the same curve system.

- Thurston used iteration on $\mathcal{T}$ in order to prove invariant structures ([Mc90]), for example the existence of a hyperbolic three-manifolds fibering over the circle. In their paper [KM96], Kaimanovich-Masur deduced that subgroups of $M C G$ cannot be higher rank lattices (see also [FM98]). One could hope that the present results also will find application.

- Our approach might be relevant for the study of random walks on $O u t\left(F_{n}\right)$; the problem of extending the Kaimanovich-Masur theory is a well-known research problem.

- As already indicated, one could moreover hope that our weak Wolff-Denjoy analog could shed some light on the dynamics of rational maps in complex dynamics, the so-called Thurston obstruction theorem also leads to considering the iteration of a holomorphic self-map such as Thurston's pull-back maps. In this latter context the fixed point corresponds to the conformal map being combinatorially equivalent to a rational map. This is not always the case, and the conditions for checking this, i.e. verifying that the holomorphic self-map has bounded orbit is difficult in practice. The curves $\alpha$ for which $E_{P}(\alpha)=0$ might be tightly related to the Thurston obstruction.

- Does every holomorphic self-map of Teichmüller space with bounded orbit have a fixed point? A positive answer to this question would be an extension of Nielsen realization and also strengthen the analog of Theorem [ 6 with the Wolff-Denjoy theorem.

\section{Acknowledgements:}

This work was done during a wonderful term spent at the Institute Mittag-Leffler. Special thanks are due to the staff for their very efficient and kind assistance. For this paper I was inspired by the recent progress in the understanding of horofunctions by Walsh, Miyachi, and Liu-Su. I got the idea of generalizing Theorem 1 to random products while preparing a presentation of [T88] at a reading seminar in Geneva and while keeping in mind similiar questions that Margulis suggested to me years ago. In addition, I am grateful for various discussions with Moon Duchin, Sebastian Hensel, Vadim Kaimanovich, Athanase Papadopoulos, John Smillie, and Giulio Tiozzo.

\section{Proof of Theorem 3}

Let $(\Omega, p)$ be a standard Borel space with $p(\Omega)=1$ and $T: \Omega \rightarrow \Omega$ an ergodic measure preserving transformation. Assume that $g: \Omega \rightarrow \mathrm{Homeo}^{+}(M) \rightarrow M C G(M)$ is a measurable 
map and let

$$
Z_{n}(\omega)=g(\omega) g(T \omega) \ldots g\left(T^{n-1} \omega\right)
$$

which is called an ergodic cocycle. Notice here that we have shifted the order, so that in the terminology of the theorem, $f_{\dot{n}}=Z_{n}^{-1}$ and the $g_{i}=\left(g\left(T^{i-1} \omega\right)\right)^{-1}$. A random walk on $M C G$ is the special case when the increments $g\left(T^{i} \omega\right)$ are assumed to be independent and identically distributed (this is precisely the case when $(\Omega, p)$ is a product space and $T$ the shift).

For $x \in \mathcal{T}$ denote by $l_{x}(\alpha)$ the minimal length in its isotopy class in the hyperbolic metric $x$ (or more precisely the class of isometric metrics all mutually isotopic). Let us recall Thurston's asymmetric metric ([T86]),

$$
L(x, y)=\log \sup _{\alpha \in \mathcal{S}} \frac{l_{y}(\alpha)}{l_{x}(\alpha)} .
$$

It is easy to see that $L$ verifies the triangle inequality, and it also true that it seperates points although this is non-trivial. Therefore $L$ satisfies all the axioms for a metric except the symmetry, which indeed fails except in very special cases of surfaces with symmetries. The triangle inequality reads

$$
L(x, z) \leq L(x, y)+L(y, z) .
$$

The metric is clearly invariant: $L(g x, g y)=L(x, y)$ for $g \in M C G$. The topology induced by $L$ coincides with the usual one, see [PT07.

Fix a base point $x_{0} \in \mathcal{T}$. We will assume that

$$
\int_{\Omega} L\left(g(\omega) x_{0}, x_{0}\right)+L\left(x_{0}, g(\omega) x_{0}\right) d p(\omega)<\infty,
$$

in which case we refer to $f_{n}$ as an integrable ergodic cocycle, or informally as a random product of mapping class elements.

One has the following subadditivity property:

$$
\begin{gathered}
\left.L\left(f_{n+m}(\omega) x_{0}, x_{0}\right) \leq L\left(f_{n}(\omega) f_{m}\left(T^{n} \omega\right) x_{0}, f_{n}(\omega) x_{0}\right)\right)+L\left(f_{n}(\omega) x_{0}, x_{0}\right) \\
=L\left(f_{m}\left(T^{n} \omega\right) x_{0}, x_{0}\right)+L\left(f_{n}(\omega) x_{0}, x_{0}\right) .
\end{gathered}
$$

From the subadditive ergodic theorem of Kingman one then knows that for a.e. $\omega$ the following limit exists (the finite non-negative value being independent of $\omega$ by the ergodicity assumption):

$$
l:=\lim _{n \rightarrow \infty} \frac{1}{n} L\left(f_{n}(\omega) x_{0}, x_{0}\right) .
$$

We now introduce concepts from the work of Walsh W11 which gave a crucial inspiration for the present paper. It will be important to consider functions $h$ in the so-called horofunction compactification of $\mathcal{T}$, that is, for $\mu \in P M F$

$$
h_{\mu}(x)=\log \sup _{\alpha} \frac{i(\mu, \alpha)}{l_{x}(\alpha)}-\log \sup _{\beta} \frac{i(\mu, \beta)}{l_{x_{0}}(\beta)},
$$

(notice that it is well-defined for projective equivalence classes of measured foliations $\mu$ ) and for $x_{n} \rightarrow \mu$ in the Thurston compactification one has

$$
h_{\mu}(x)=\lim _{n \rightarrow \infty} L\left(x, x_{n}\right)-L\left(x_{0}, x_{n}\right) .
$$


These functions together with $h_{z}(x)=L(x, z)-L\left(x_{0}, z\right)$ constitute a compact space $H$ homeomorphic to Thurston's compactification as proved by Walsh. Notice that

$$
h_{z}(x)=L(x, z)-L\left(x_{0}, z\right) \leq L\left(x, x_{0}\right)
$$

by the triangle inequality.

For $f \in M C G$ and $h$ as above let $F(g, h)=-h\left(g^{-1} x_{0}\right)$. We note the following cocycle property:

$$
\begin{aligned}
& F\left(g_{1}, g_{2} h\right)+F\left(g_{2}, h\right)=-\left(g_{2} \cdot h\right)\left(g_{1}^{-1} x_{0}\right)-h\left(g_{2}^{-1} x_{0}\right) \\
= & -h\left(g_{2}^{-1} g_{1}^{-1} x_{0}\right)+h\left(g_{2}^{-1} x_{0}\right)-h\left(g_{2}^{-1} x_{0}\right)=F\left(g_{1} g_{2}, h\right) .
\end{aligned}
$$

Note that moreover

$$
L\left(g x_{0}, x_{0}\right)=-L\left(g^{-1} x_{0}, g^{-1} x_{0}\right)+L\left(x_{0} g^{-1} x_{0}\right)=\max _{h \in H} F(g, h),
$$

as follows from the triangle inequality. Following KaL06, see also KaL11, one introduces the skew product system $\bar{T}: \Omega \times H \rightarrow \Omega \times H$ by $\bar{T}(\omega, h)=\left(T \omega, g^{-1}(\omega) h\right)$ and checks that with $\bar{F}(\omega, h):=F\left(g(\omega)^{-1}, h\right)$ one has that

$$
F\left(f_{n}(\omega)^{-1}, h\right)=\sum_{i=0}^{n-1} \bar{F}\left(\bar{T}^{i}(\omega, h)\right) .
$$

Moreover, we have $\left|F\left(g^{1}(\omega), h\right)\right| \leq \max \left\{L\left(x_{0}, g(\omega) x_{0}\right), L\left(g(\omega) x_{0}, x_{0}\right)\right\}$ so $F$ is integrable (notice here again a small difference due to the asymmetric nature of $L$ ). From this point on, the proof runs as in the references mentioned, that is, a special measure is constructed which accounts for the drift as well as projecting to the original one. Birkhoff's ergodic theorem is applied and a measurable section is taken. We get that for a.e. $\omega$ there is an $h=h^{\omega}$ such that

$$
\lim _{n \rightarrow \infty}-\frac{1}{n} h\left(Z_{n} x_{0}\right)=l
$$

If $l>0$, which is the nontrivial case, it is immediate that $h$ is a boundary point, and hence by Walsh's theorem cited above, it is of the form $h_{\mu}$ for some $\mu \in P M F$. This means that for every $\epsilon>0$ there is an $N$ such that for all $n>N$ one has

$$
\log \sup _{\alpha} \frac{i(\mu, \alpha)}{l_{Z_{n} x_{0}}(\alpha)}-\log \sup _{\beta} \frac{i(\mu, \beta)}{l_{x_{0}}(\beta)} \leq-(l-\epsilon) n .
$$

Letting $C_{\mu}^{-1}=\sup \frac{i(\mu, \beta)}{l_{x_{0}}(\beta)}$ we then obtain

$$
\sup _{\alpha} \frac{i(\mu, \alpha)}{l_{Z_{n} x_{0}}(\alpha)} \leq C_{\mu}^{-1} e^{-(l-\epsilon) n}
$$

which leads to that for every $\alpha$ we have

$$
l_{Z_{n} x_{0}}(\alpha) \geq C_{\mu} i(\mu, \alpha) e^{(l-\epsilon) n} .
$$

On the other hand, one knows (see for example [CR07, LPST10]) that for any two points $x$ and $y$ in the thick part of Teichmüller space up to an additive constant $L(x, y) \asymp L(y, x)$, thanks to the symmetry of the Teichmüller metric. Therefore, since the orbit $Z_{n} x_{0}$ stays in 
the thick part (as it does not move in the moduli space), we have from the subadditive ergodic theorem mentioned above that for all sufficiently large $n$,

$$
L\left(x_{0}, Z_{n} x_{0}\right)=\log \sup _{\alpha} \frac{l_{Z_{n} x_{0}}(\alpha)}{l_{x_{0}}(\alpha)} \leq(l+\epsilon) n .
$$

In view of these two inequalities we have for every $\alpha \in \mathcal{S}$ with $i(\mu, \alpha)>0$ that

$$
l_{Z_{n} x_{0}}(\alpha)^{1 / n} \rightarrow \lambda:=e^{l} .
$$

Note that $l_{x_{0}}\left(f_{n} \alpha\right)=l_{Z_{n} x_{0}}(\alpha)$ and that since $M$ is compact from the point of view of exponential growth every Riemannian metric is equivalent, so we can replace $x_{0}$ with $\rho$. This finishes the proof of Theorems 2 and 3 . We also remark is that if we do not assume ergodicity, by ergodic decomposition, there is no change other than that the "Lyapunov exponent" $\lambda$ is now random and not necessarily constant. A final comment is that $M$ could be allowed to have punctures, except possibly for restricting to Riemannian metrics equivalent to hyperbolic metrics.

\section{Additional arguments in the random walk case}

Assume that $\nu$ is a probability measure on $M C G$ of finite first moment (here meaning that the cocycle is integrable) and whose support generates a non-elementary subgroup. A nonelementary subgroup is by definition a subgroup which do not fix any finite set in $\mathcal{P} \mathcal{M F}$. It is shown in KM96 that this is equivalent to that there are at least two pseudo-Anosovs with disjoint fixed point sets. In particular it implies that the subgroup is non-amenable. Kaimanovich and Masur KM96 established that random walks a.s. converge to points in the set of uniquely ergodic foliations of $\mathcal{P} \mathcal{M F}$. Moreover, they show that the measure that this convergence gives rise to, the harmonic or hitting measure, is the unique $\nu$-stationary measure. In my work with Ledrappier we construct special $\nu$-stationary measures in a general setting, see [KaL11, Theorem 18]. By uniqueness, this measure is hence the same as the hitting one and by the remark towards the end of the proof of [KaL11, Theorem 18] we get the same conclusion (11) above. Now the random foliations $\mu$ are uniquely ergodic, so $i(\mu, \alpha)>0$ for any $\alpha \in \mathcal{S}$. Finally, $\lambda>1$ because $l>0$ which in turn is a consequence of entropy theory, including a well-known inequality of Guivarc'h, and that a non-elementary subgroup is non-amenable as well as the fact that $\mathcal{T}$ has at most exponential growth as established in [KM96], see for example [K00] for this type of arguments. This proves Corollary 4 ]

\section{Proof of Theorem 5}

Recall Kerckhoff's formula for the Teichmüller distance:

$$
d(x, y)=\frac{1}{2} \log \sup _{\alpha \in \mathcal{S}} \frac{\operatorname{Ext}_{x}(\alpha)}{\operatorname{Ext}_{y}(\alpha)},
$$

where Ext denotes extremal length and defined in the introduction. Royden showed that this metric coincides with the Kobayashi metric associated to the complex structure of $\mathcal{T}$. This implies that holomorophic maps are 1-Lipschitz in this metric, see [E01 for an update on finer contraction properties. Gardiner and Masur introduced in GM91 a compactification analogous to the Thurston compactification but using extremal lengths instead of hyperbolic lengths. 
Fix a point $x_{0} \in \mathcal{T}$. Let

$$
E_{x}(\alpha)=\frac{\operatorname{Ext}_{x}(\alpha)^{1 / 2}}{K_{x}^{1 / 2}},
$$

where $K_{x}$ is the quasi-conformal dilation of the Teichmüller map from $x_{0}$ to $x$. Miyachi Mi08] noted that $E$ extends continuously to a function defined on the Gardiner-Masur compactification $\overline{\mathcal{T}}^{G M}$ of $\mathcal{T}$.

Let $f: \mathcal{T} \rightarrow \mathcal{T}$ be a holomorphic self-map of Teichmüller space. Define

$$
l=\lim _{n \rightarrow \infty} \frac{1}{n} d\left(f^{n} x_{0}, x_{0}\right)
$$

which exists by the 1 -Lipschitz property and subadditivity. Clearly, $0 \leq l<\infty$. Moreover, for any point $P \in \overline{\mathcal{T}}^{G M}$ define following Liu and $\mathrm{Su}$

$$
h_{P}(x)=\log \sup _{\beta} \frac{E_{P}(\beta)}{E_{x}(\beta)^{1 / 2}}-\log \sup _{\alpha} \frac{E_{P}(\alpha)}{E x t_{x_{0}}(\alpha)^{1 / 2}} .
$$

We set for short

$$
Q(P)=\sup _{\alpha} \frac{E_{P}(\alpha)}{E_{x x_{0}}(\alpha)^{1 / 2}} .
$$

Given a sequence $\epsilon_{i} \searrow 0$ we set $b_{i}(n)=d\left(f^{n} x_{0}, x_{0}\right)-\left(l-\epsilon_{i}\right) n$. Since these numbers are unbounded, we can find a subsequence such that $b_{i}\left(n_{i}\right)>b_{i}(m)$ for any $m<n_{i}$ and by sequential compactness we may moreover assume that $f^{n_{i}}\left(x_{0}\right) \rightarrow P \in \overline{\mathcal{T}}^{G M}$, cf. [Ka01].

By a result of Liu and Su [LS10] identifying the horoboundary compactification of $(\mathcal{T}, d)$ with the Gardiner-Masur compactification (in particular showing that the latter is metrizable) we have for any $k \geq 1$ that

$$
\begin{gathered}
h_{P}\left(f^{k} x_{0}\right)=\lim _{i \rightarrow \infty} d\left(f^{k} x_{0}, f^{n_{i}} x_{0}\right)-d\left(x_{0}, f^{n_{i}} x_{0}\right) \\
\leq \liminf _{i \rightarrow \infty} d\left(x_{0}, f^{n_{i}-k} x_{0}\right)-d\left(x_{0}, f^{n_{i}} x_{0}\right) \\
\leq \liminf _{i \rightarrow \infty} b_{i}\left(n_{i}-k\right)+\left(l-\epsilon_{i}\right)\left(n_{i}-k\right)-b_{i}\left(n_{i}\right)-\left(l-\epsilon_{i}\right) n_{i} \\
\leq \liminf _{i \rightarrow \infty}-\left(l-\epsilon_{i}\right) k=-l k .
\end{gathered}
$$

This means in terms of extremal lengths that

$$
\left(\sup _{\beta} \frac{E_{P}(\beta)}{E_{x_{f^{k} x_{0}}}(\beta)^{1 / 2}}\right)^{-1} \geq Q(P)^{-1} e^{l k} .
$$

and hence for any $\beta \in \mathcal{S}$ that

$$
\operatorname{Ext}_{f^{k} x_{0}}(\beta) \geq E_{P}(\beta)^{2} Q(P)^{-2} e^{2 l k} .
$$

On the other hand, in view of Kerckhoff's formula one has an estimate from above:

$$
e^{2 d_{T}\left(f^{k} x_{0}, x_{0}\right)}=\sup _{\alpha} \frac{\operatorname{Ext}_{f^{k} x_{0}}(\alpha)}{E x t_{x_{0}}(\alpha)} \geq \frac{\operatorname{Ext}_{f^{k} x_{0}}(\beta)}{\operatorname{Ext}_{x_{0}}(\beta)} .
$$

In particular, provided $E_{P}(\beta)>0$, the two estimates imply that

$$
\operatorname{Ext}_{f^{k} x_{0}}(\beta)^{1 / n} \rightarrow e^{2 l}
$$

which by letting $\lambda=e^{2 l}$ and setting $x=x_{0}$ finishes the proof of Theorem 5 ,

Note here that $l$, or $\lambda$, are independent of $x$, while $P$ might depend mildly on the point $x$. 


\section{Additional arguments concluding the proof of Theorem 6}

Suppose that the orbit is unbounded, then by a theorem of Calka, the orbits tends to infinity, see [Ka05]. Moreover, $l$ is defined as above and we suppose that $P$ is a uniquely ergodic point. From Miyachi [Mi08, Proposition 5.1] it then follows that we have that this subsequence also converges to $P$ in the Thurston boundary. Then we know from combining Theorem 11 with Corollary 45 in Ka05 that the whole forward orbit must converge to $P$ (and hence in both compactifications).

In the notation above, we finally have

$$
\begin{gathered}
h_{P}(f(y))=\lim _{i \rightarrow \infty} d\left(f(y), f^{n_{i}} x_{0}\right)-d\left(x_{0}, f^{n_{i}} x_{0}\right) \\
\leq \liminf _{i \rightarrow \infty} d\left(y, f^{n_{i}-1} x_{0}\right)-d\left(x_{0}, f^{n_{i}} x_{0}\right) \\
\leq \liminf _{i \rightarrow \infty} d\left(y, f^{n_{i}-1} x_{0}\right)-d\left(x_{0}, f^{n_{i}-1} x_{0}\right)-\left(l-\epsilon_{i}\right) \\
=h_{P}(y)-l
\end{gathered}
$$

From this we may conclude Theorem 6 as in the previous proof (note that this time we have $\log Q(P)$ on both sides, hence this cancels out).

In the bounded orbit case, we recall that if $f$ is a mapping class then there is a fixed point by a theorem of Nielsen, if $f$ is a strict contraction then by a simple argument due to Edelstein the orbit converges towards the unique fixed point. However, in general we do not know this. We end by remarking that [Mi08, Proposition 5.1] also in the reducible case (i.e. unbounded orbits but $P$ not uniquely ergodic) gives information on the possible boundary limit points of the orbit in terms of intersections with $P$.

\section{References}

[A98] Arnold, Ludwig, Random dynamical systems. Springer Monographs in Mathematics. Springer-Verlag, Berlin, 1998. xvi +586 pp.

[B78] Bers, Lipman An extremal problem for quasiconformal mappings and a theorem by Thurston. Acta Math. 141 (1978), no. 1-2, 73-98.

[CR07] Choi, Young-Eun; Rafi, Kasra Comparison between Teichmüller and Lipschitz metrics. J. Lond. Math. Soc. (2) 76 (2007), no. 3, 739-756

[D05] Duchin, Moon; Thin triangles and a multiplicative ergodic theorem for Teichmüller geometry, http://front.math.ucdavis.edu/math.GT/0508046

[E01] Earle, Clifford J. Schwarz's lemma and Teichmüller contraction. Complex manifolds and hyperbolic geometry (Guanajuato, 2001), 79-85, Contemp. Math., 311, Amer. Math. Soc., Providence, RI, 2002.

[FMa12] Farb, Benson; Margalit, Dan A primer on mapping class groups. Princeton Mathematical Series, 49. Princeton University Press, Princeton, NJ, 2012. xiv +472 pp.

[FM98] Farb, Benson; Masur, Howard Superrigidity and mapping class groups. Topology 37 (1998), no. 6, 1169-1176. 
[Ga09] Gadre, V, Harmonic measures for distributions with finite support on the mapping class group are singular, (2009), http://arxiv.org/abs/0911.2891

[GM91] Gardiner, F, and Masur, M, Extremal length geometry of Teichmüller space, Complex Variable 16 (1991), 23-41.

[FLP79] Fathi, A, Laudenbach, F, and Poénaru, V. Travaux de Thurston sur les surfaces. Astérisque, 66-67. Société Mathématique de France, Paris, 1979. 284 pp.

[K00] Kaimanovich, Vadim A. The Poisson formula for groups with hyperbolic properties. Ann. of Math. (2) 152 (2000), no. 3, 659-692.

[KM96] Kaimanovich, Vadim A.; Masur, Howard The Poisson boundary of the mapping class group. Invent. Math. 125 (1996), no. 2, 221-264.

[Ka01] Karlsson, Anders Non-expanding maps and Busemann functions. Ergodic Theory Dynam. Systems 21 (2001), no. 5, 1447-1457.

[Ka05] Karlsson, Anders On the dynamics of isometries. Geom. Topol. 9 (2005), 2359-2394.

[KaL06] Karlsson, Anders; Ledrappier, François On laws of large numbers for random walks. Ann. Probab. 34 (2006), no. 5, 1693-1706,

[KaL11] Karlsson, Anders; Ledrappier, François Noncommutative ergodic theorems. Geometry, rigidity, and group actions, 396-418, Chicago Lectures in Math., Univ. Chicago Press, Chicago, IL, 2011,

[LPST10] Liu, Lixin; Papadopoulos, Athanase; Su, Weixu; Théret, Guillaume, Length spectra and the Teichmüller metric for surfaces with boundary. Monatsh. Math. 161 (2010), no. 3, 295-311

[LPST11] Liu, Lixin; Papadopoulos, Athanase; Su, Weixu; Théret, Guillaume, On the classification of mapping class actions on Thurston's asymmetric metric, arXiv:1110.3601v1

[LS10] Liu, Lixin, Su, Weixu, The horofunction compactification of Teichmüller metric, In: Handbook of Teichmüller theory, (A. Papadopoulos ed.) Volume IV, IRMA Lecture Notes in Mathematics and Theoretical Physics Vol. 19, EMS Publishing House, Zürich, 2013 (to appear)

[Ma11] Maher, Joseph Random walks on the mapping class group, Duke Math. J. 156 (2011), no. 3, 429-468.

[M95] Masur, Howard, Random walks on Teichmuller space and the mapping class group. J. Anal. Math. 67 (1995), 117-164

[Mc90] McMullen, C. Iteration on Teichmüller space. Invent. Math. 99 (1990) 425-454.

[Mi08] Miyachi, H, Teichmüller rays and the Gardiner-Masur boundary of Teichmüller space, Geom. Dedicata 137 (2008), 113-141.

[Mi11] Miyachi, H., Teichmüller space has non-Busemann points, arXiv:1105.307v1, 2011

[N] Nielsen, Jakob Untersuchungen zur Topologie der geschlossenen zweiseitigen Flächen. Acta Math. 50 (1927) 189-358; 53 (1929) 1-76; 58 (1932) 87-167; 75 (1943) 23-115. 
[N44] Nielsen, Jakob Surface transformation classes of algebraically finite type. Danske Vid. Selsk. Math.-Phys. Medd. 21, (1944). no. 2, 89 pp.

[PT07] Papadopoulos, Athanase; Théret, Guillaume, On the topology defined by Thurston's asymmetric metric. Math. Proc. Cambridge Philos. Soc. 142 (2007), no. 3, 487-496

[Pi01] Pilgrim, Kevin M. Canonical Thurston obstructions. Adv. Math. 158 (2001) 154-168.

[S11] Selinger, Nikita, Thurston's pullback map on the augmented Teichmüller space and applications, Invent. Math (2011)

[T88] Thurston, William P. On the geometry and dynamics of diffeomorphisms of surfaces. Bull. Amer. Math. Soc. (N.S.) 19 (1988), no. 2, 417-431

[T86] Thurston, W. Minimal stretch maps between hyperbolic surfaces. preprint, arXiv:math GT/9801039, 1986.

[Ti12] Tiozzo, Giulio, Sublinear deviation between geodesics and sample paths, May 2012

[W11] Walsh, Cormac, The horoboundary and isometry group of Thurston's Lipschitz metric, In: Handbook of Teichmüller theory, (A. Papadopoulos ed.) Volume IV, IRMA Lecture Notes in Mathematics and Theoretical Physics Vol. 19, EMS Publishing House, Zürich, 2013 (to appear)

Anders Karlsson

Section de mathématiques

Université de Genève

2-4 Rue du Lièvre

Case Postale 64

1211 Genève 4,

Suisse

e-mail: anders.karlsson@unige.ch 